\title{
Astronomical of ${ }^{\text {Joul }}$ Telescopes, Instruments, and Systems
}

\section{Methods of defining circular off-axis aspheric mirrors}

Jeong-Yeol Han

Sukmock Lee 


\title{
Methods of defining circular off-axis aspheric mirrors
}

\author{
Jeong-Yeol Han ${ }^{\mathrm{a}}$ and Sukmock Lee $\mathrm{e}^{\mathrm{b}, *}$ \\ ${ }^{a}$ Korea Astronomy and Space Science Institute, Daejeon, Republic of Korea \\ bInha University, Department of Physics, Incheon, Republic of Korea
}

\begin{abstract}
We investigated the definition for circular off-axis aspheric mirrors (COAMs). The clear opening of the COAM obtained by projecting a circular aperture on the aspheric surface does not form a plane but a curved surface. To solve this problem, we propose to use a plane to cut the aspheric surface to obtain a COAM. The COAM has three characteristics: (i) an elliptical opening; (ii) the major axis of the opening lies on the meridional plane containing the original optical axis of the aspheric surface and the minor axis lies on the sagittal plane perpendicular to the meridian plane; and (iii) it is not deepest at the center of the ellipse. The third characteristic suggests two methods for defining the COAM using a plane: (i) with the center of the elliptical opening as a reference and (ii) with the deepest point of the mirror as a reference. All the formulas required to obtain the desired COAM using the two methods are presented. () The Authors. Published by SPIE under a Creative Commons Attribution 3.0 Unported License. Distribution or reproduction of this work in whole or in part requires full attribution of the original publication, including its DOI. [DOI: 10.1117/1.JATIS.4.4.044002]
\end{abstract}

Keywords: geometric optics; optical testing; circular off-axis aspheric mirror; reference optical axis.

Paper 18039 received Jun. 1, 2018; accepted for publication Sep. 26, 2018; published online Oct. 19, 2018.

\section{Introduction}

An off-axis aspheric mirror is a well-established form for largescale telescope mirrors, and many of the recently developed telescopes employ a large number of segmented off-axis aspheric mirrors. Some examples include the Giant Magellan Telescope (GMT) ${ }^{1,2}$ the Thirty Meter Telescope (TMT), ${ }^{3}$ the European Extremely Large Telescope (EELT), ${ }^{4}$ the James Webb Space Telescope (JWST), ${ }^{5}$ and the Daniel K. Inouye Solar Telescope. ${ }^{6}$

In addition to the radius of curvature $R$ and conic constant $K$ to represent the aspheric surface, each segment mirror requires two additional parameters: (i) the size of the mirror and (ii) the distance from the reference optical axis (ROA) of the aspheric surface to the center of the mirror. ${ }^{7}$ Although it is easy to understand the meanings of the two parameters from a simple linguistic point of view, there are practical ambiguities that can cause problems. If the shape of the mirror is not an exact circle, it is unclear whether the size denotes the size of the surface on which the light is actually reflected or whether it denotes the longest size or the shortest size. Furthermore, for most lenses or mirrors of aspheric surfaces in rotational symmetry, various terms such as the center of the opening, vertex, center of curvature, deepest point, or the optical axis all refer to the same element. The ROA is the normal line at the vertex of the aspheric surface that passes through the center of the surface or opening. It is the deepest point at the center of the opening. For off-axis aspheric mirrors, all of the above terms might convey different meanings. Thus, the additional parameters are only abstract unless sufficiently accurate definitions are provided. We believe such a detailed treatment is not currently available.

The above-mentioned large astronomical telescopes commonly have one large mirror, which is composed of a number of segment mirrors but can be roughly divided into two categories: (i) TMT, EELT, and JWST are one category, in which the segment mirrors are all connected, and (ii) GMT is another category, in which all the elements are separated. For the mirrors to

*Address all correspondence to: Sukmock Lee, E-mail: smlee@inha.ac.kr be connected, not only must the shape of each mirror be correct but also the curved surface of the mirror must be continuous at all the connecting sides, and it is hard to imagine alternative ways of satisfying these obvious requirements for the first category of mirrors. By contrast, the condition of continuity between the seven primary segments of the GMT apart from each other is not as obvious as for those in the first category. ${ }^{8,9}$ In addition, the circular shape of each segment mirror for the GMT raises some questions: how precisely circular is the opening of the mirror and how flat is the opening. These points were unclear enough to stimulate us to conduct the present work.

In order to answer the numerous unclear points, we first tested a way of acquiring a circular off-axis aspheric mirror (COAM) by simple projection, in which a circular aperture was simply projected on an aspheric surface. The results show that the COAM opening is not a plane but a curved surface, as described in Sec. 2. This result has caused considerable difficulty in establishing shapes and related terms for the COAM, and the necessity of developing methods to define the COAM has been raised. Thus, we propose a method to define the COAM by cutting the aspheric surface with a plane to make the COAM opening a plane. In Sec. 3, we derived the relations between the plane and the COAM, which can be reversed to find the characteristics of the plane for the desired COAMs. In Sec. 4, the depth of the COAM mirror is analyzed, and two conditions for defining the plane are identified. In Secs. 5 and 6, the characteristics of the COAM, defined by the two definitions, are described in detail.

\section{Nonplanar Opening of a COAM Created by Projecting Circular Aperture}

The sag or $z$-component of the aspheric surface with conic constant $K$ and radius of curvature $R$ as a function of $(x, y)$ is expressed as follows:

$$
z(x, y)=\frac{R-\sqrt{R^{2}-(K+1)\left(x^{2}+y^{2}\right)}}{K+1},
$$




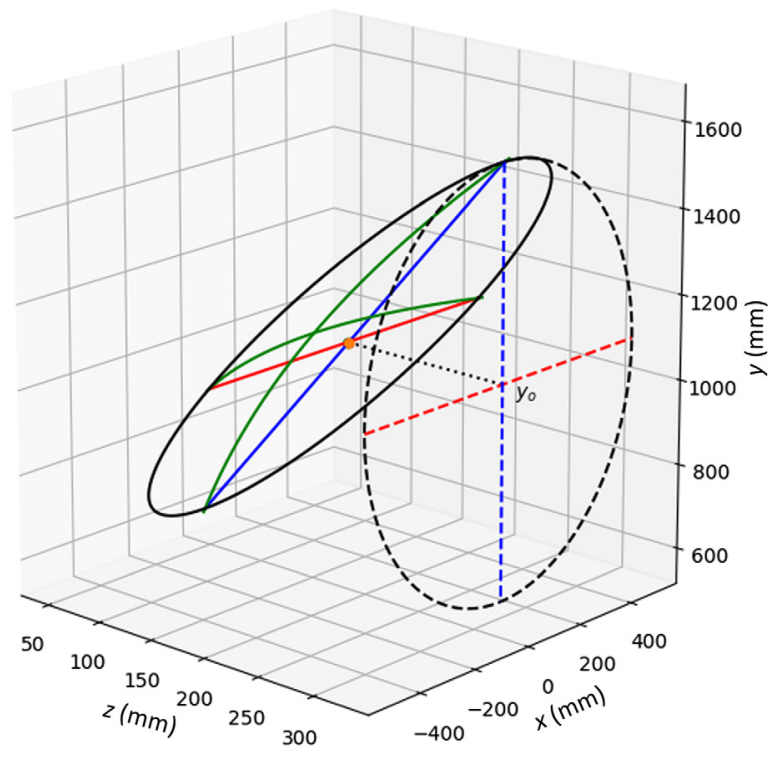

Fig. $13-D$ plot of COAM created by projecting a circular aperture.

or

$x^{2}+y^{2}=2 R z-(K+1) z^{2}$.

In the absence of a standard way of dealing with the COAM, one easy way to define a COAM of a desired size is to project a circular opening of the required size horizontally to the aspheric surface, as shown in Fig. 1. The corresponding equation for the opening can be expressed as follows:

$x^{2}+\left(y-y_{0}\right)^{2}=R_{c}^{2}$,

where $y_{o}$ is the distance from the ROA, which is the $z$-axis, to the center of the opening, and $R_{\mathrm{c}}$ is the semidiameter of the opening, the desired size. The circular opening is assumed to be in a plane perpendicular to the ROA. If we project this circular opening on the aspheric surface, then the projected intercepts are expressed as follows:

$\mathrm{z}=\frac{R-\sqrt{R^{2}-(K+1)\left(R_{c}^{2}-y_{0}^{2}+2 y_{0} y\right)}}{K+1}$.

Figure 1 shows the schematic of a COAM with two $z$-components in green indicating the depth in two orthogonal directions and with round intercepting points in black indicating the clear opening. The circular aperture is depicted by a dashed line. The blue vertical and red horizontal dashed lines on the circular aperture, respectively, pass through the center of the aperture, which is denoted as $y_{0}$. The blue solid line on the COAM is the connecting line between the two projected points of both ends of the blue dashed line of the aperture and forms a meridional plane with a corresponding green depth line. Among many meridional planes, the meridional plane becomes the reflection symmetric plane for the COAM. Similarly, the red solid line is also the connecting line between two projected points of both ends of the red dashed line. The example shown in Fig. 1 was generated for the set of numerical values $R=4000 \mathrm{~mm}$, $K=-0.7, R_{\mathrm{c}}=500 \mathrm{~mm}$, and $y_{0}=1100 \mathrm{~mm}$.

The opening looks flat at first sight. However, it is not a plane but a curved surface. We attempted to demonstrate the amount of deviation from a plane in two different ways. First, we considered the shortest distance between two blue and red solid skew lines in Fig. 1. The horizontal line can be represented as the point $\left(0, y_{0}, z_{0}\right)$ on the basis of the rotational symmetry property of the COAM, where $z_{0}$ is expressed as follows:

$z_{0}=\frac{R-\sqrt{R^{2}-(K+1)\left(y_{0}^{2}+R_{c}^{2}\right)}}{K+1}$.

If the top and bottom points are $\left(0, y_{\mathrm{T}}, z_{\mathrm{T}}\right)$ and $\left(0, y_{\mathrm{B}}, z_{\mathrm{B}}\right)$, respectively, where

$\left\{\begin{array}{l}y_{T}=y_{0}+R_{C} \\ y_{B}=y_{0}-R_{C} \\ z_{T}=\frac{R-\sqrt{R^{2}-(K+1)\left(y_{0}+R_{C}\right)^{2}}}{K+1}, \\ z_{B}=\frac{R-\sqrt{R^{2}-(K+1)\left(y_{0}-R_{C}\right)^{2}}}{K+1}\end{array}\right.$

the line connecting the two points can be expressed as $a z+b y+c=0$, where

$\left\{\begin{array}{l}a=y_{T}-y_{B} \\ b=z_{B}-z_{T} \\ c=y_{B} z_{T}-y_{T} z_{B}\end{array}\right.$.

The shortest distance between the two lines is then given as in Ref. 10:

$D=\frac{\left|a z_{0}+b y_{0}+c\right|}{\sqrt{a^{2}+b^{2}}}$,

and is nonzero for $-1<K<0$, indicating that the opening of the COAM is clearly not a plane. For the numerical COAM shown in Fig. 1, the shortest distance was $0.712 \mathrm{~mm}$.

The second endeavor is to visualize the gap between the two lines, as shown in Fig. 2. Although Eq. (4) clearly shows the relation between the independent variable $y$ and the dependent variable $z$, the analysis was not easy. Equation (4) can be expanded as a Taylor series about $y=y_{0}$, indicating the sag as a polynomial. The fact that the opening looks flat indicates that the first-order term of the polynomial is dominant. Thus, to emphasize the curved opening, the first-order term is removed

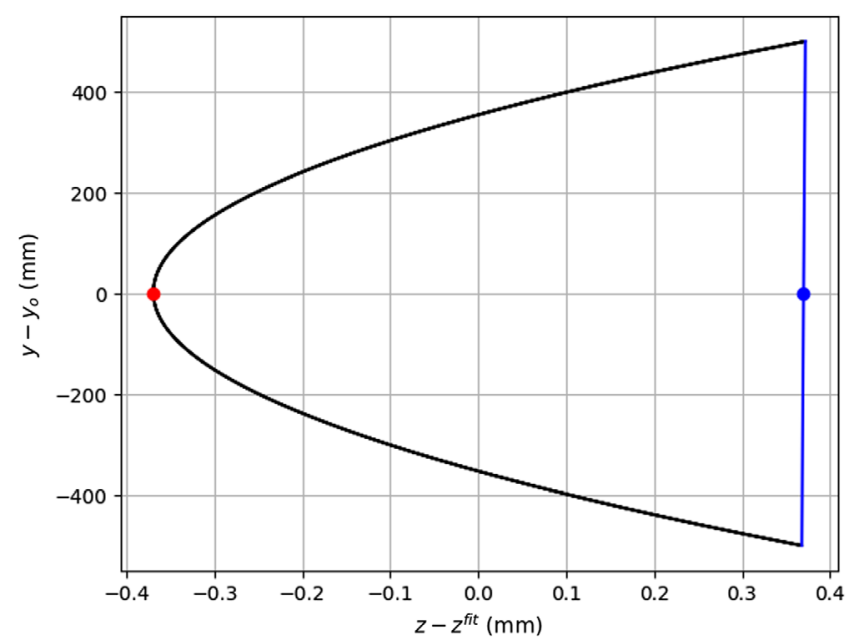

Fig. 2 Numerical result of Eq. (4) with the linear term removed. 
by employing numerical curve-fitting analysis. If the opening of the COAM was a plane, then the sag would be zero for all $y$ values after its removal. Figure 2 shows the numerical result of Eq. (4) for the COAM shown in Fig. 1, and the opening cross-section of Eq. (4) with the linear term removed clearly demonstrates the curved opening.

After a thorough investigation, it is now clear that the curved opening is a result of the original aspheric surface, whether the COAMs are connected to each other or not. That is, the seven segments of GMT can be simply considered as a set of continuous segmented mirrors with the interconnecting segments removed.

However, despite the fact that the distance between the centers of the horizontal and vertical lines is longer than the shortest distance between the lines for the example shown in Fig. 1, the curved opening reveals several important factors if the segment was obtained by projecting a circular aperture: the opening is not circular and is not flat but curved several tenths of millimeters upward toward both the top and bottom parts.

Further study revealed the possibility that the nonplanar opening can become flat if the projecting aperture is not circular. This possibility motivated the work of finding a way to define COAMs.

\section{Aspheric Surface, a Plane, and an Off-Axis Aspheric Segment}

Instead of analyzing the detailed shape of the opening for the COAM obtained by projection, we propose a way to define the COAM. Let us consider the same aspheric surface, whose section in the $y z$-plane is shown in Fig. 3. The $z$-axis and the origin of the coordinate system correspond to the ROA and the vertex of the surface, respectively. To begin with the planar opening for the COAM, we used a plane. The red line, shown in Fig. 3, depicts the plane intersecting with the aspheric surface. The angle between the plane and the $x z$-plane is $\theta$, and the plane intercepts the $x y$-plane at the point (or line to be exact) $y=y_{\mathrm{C}}$. Thus, we define a COAM as a part of the aspheric surface cut-off by the plane so that the clear opening of the COAM is evidently a plane to begin with.

Although the original $x y z$-system is convenient to present the aspheric surface, it is neither convenient to analyze the depth property of the COAM nor it is intuitive. Thus, a $x u v$-coordinate

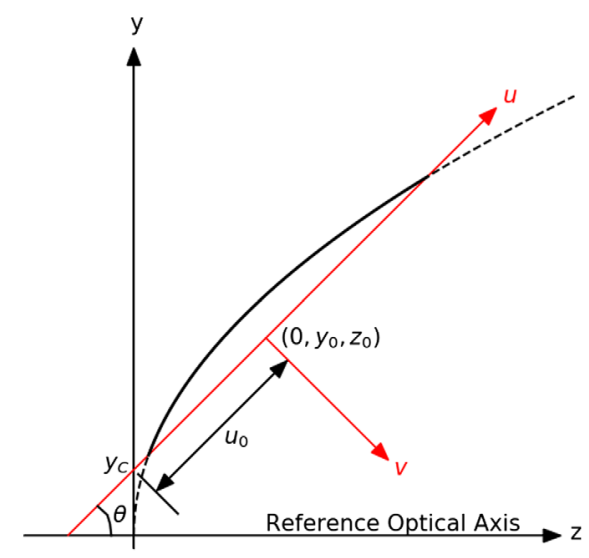

Fig. 3 Schematic diagram of an aspheric surface in a dashed line with respect to the coordinate system, whose origin and $z$-axis coincide with the vertex and rotational axis of the surface, respectively. The coordinate system of $(x, u, v)$ is depicted in red. system is introduced, as depicted in the red lines in Fig. 3. The $x$-axis is the same; the $u$-axis is perpendicular to the $x$-axis and lies on the plane, cutting the aspheric surface to form a COAM; and the $v$-axis is perpendicular both axes. The origin of the system is at the center of the clear opening of the COAM, and the coordinates are $\left(0, y_{0}, z_{0}\right)$ with respect to the original $x y z$-system. It is at a distance $u_{0}$ from the $y$-intercept $y_{\mathrm{C}}$, where $\left(y_{0}-y_{\mathrm{C}}\right)^{2}+z_{0}^{2}=\mathrm{u}_{0}^{2}$. The transformation matrix between the two systems can be expressed as follows:

$$
\left(\begin{array}{c}
x \\
y-y_{C} \\
z
\end{array}\right)=\left(\begin{array}{ccc}
1 & 0 & 0 \\
0 & \sin \theta & -\cos \theta \\
0 & \cos \theta & \sin \theta
\end{array}\right)\left(\begin{array}{c}
x \\
u+u_{0} \\
v
\end{array}\right) .
$$

Because the intercept points between the plane and the aspheric surface lie on the plane $v=0$, they are expressed as follows:

$$
\left\{\begin{array}{l}
x=x \\
y=\sin \theta\left(u+u_{0}\right)+y_{C} \\
z=\cos \theta\left(u+u_{0}\right)
\end{array}\right.
$$

Then after substitution of Eq. (10), Eq. (2) can be rewritten as follows:

$$
\begin{aligned}
x^{2}+\left(\sin \theta\left(u+u_{0}\right)+y_{C}\right)^{2}= & 2 R \cos \theta\left(u+u_{0}\right) \\
& -(K+1) \cos ^{2} \theta\left(u+u_{0}\right)^{2},
\end{aligned}
$$

and after some algebra, Eq. (11) can be converted into the equation of an ellipse as follows:

$$
\left(\frac{x}{R_{x}}\right)^{2}+\left(\frac{u}{R_{u}}\right)^{2}=1,
$$

where

$$
\left\{\begin{array}{l}
u_{0}=\frac{R \cos \theta-y_{C} \sin \theta}{K \cos ^{2} \theta+1} \\
R_{x}=\sqrt{K \cos ^{2} \theta+1} R_{u} \\
R_{u}=\frac{\cos \theta}{K \cos ^{2} \theta+1} \sqrt{R^{2}-2 y_{C} \mathrm{R} \tan \theta-y_{C}^{2}(K+1)}
\end{array} .\right.
$$

For $0<K<-1$, the ratio $R_{\mathrm{x}} / R_{\mathrm{u}}$ is always $<1$, so that $R_{\mathrm{x}}$ represents the minor and $R_{\mathrm{u}}$ represents the major semidiameter of the elliptical opening. That is, the opening of the COAM cut-off from the aspheric surface by a plane is an ellipse with a horizontal minor axis and a vertical major axis. In addition, the center of the ellipse is at a distance of $u_{0}$ away from the $y$-intercept $y_{\mathrm{C}}$.

\section{Depth Profile of Off-Axis Aspheric Segment}

Equation (13) shows the characteristics of the opening of a COAM obtained by the plane of the $y$-intercept $y_{\mathrm{C}}$ and angle $\theta$, indicating the possibility of acquiring the two parameters of the plane for the desired COAM. To do so, one more parameter needs to be addressed: the depth property.

The depth of the COAM is easily identified as the $v$-value of the segment. By using Eq. (9), Eq. (2) in the $x u v$-coordinate system can be expressed as follows: 


$$
\begin{aligned}
x^{2}+ & \left(\sin \theta\left(u+u_{0}\right)-\cos \theta v+y_{C}\right)^{2} \\
= & 2 R\left(\cos \theta\left(u+u_{0}\right)+\sin \theta v\right) \\
& -(K+1)\left(\cos \theta\left(u+u_{0}\right)+\sin \theta v\right)^{2},
\end{aligned}
$$

which can be rewritten as the quadratic equation $a v^{2}-2 b v+$ $c=0$ for the depth, where

$$
\left\{\begin{aligned}
a= & \left(1+K \sin ^{2} \theta\right) \\
b= & R \sin \theta+y_{C} \cos \theta-K \cos \theta \sin \theta\left(u+u_{0}\right) \\
c= & x^{2}+\left(y_{C}\right)^{2}+2\left(y_{C} \sin \theta-R \cos \theta\right)\left(u+u_{0}\right) \\
& +\left(K \cos ^{2} \theta+1\right)\left(u+u_{0}\right)^{2}
\end{aligned}\right.
$$

Among the two solutions of the quadratic equation, the one with the negative value is the correct one. Equation (15) can be used to visualize the depth map for the region of interest for the set of values of the aspheric surface and the plane of interest. The numerical values used in Fig. 1 cannot be used directly because the values of the plane are not known yet and because the corresponding projecting circular aperture is thus not known yet. Instead, Fig. 4 shows a contour map for the depth of the COAM having $R=4000 \mathrm{~mm}, K=-0.7, y_{\mathrm{C}}=436.7 \mathrm{~mm}$, and $\theta=74.5 \mathrm{deg}$, which clearly indicates the existence of the deepest point at a different location from the center of the opening. It is easy to understand that the deepest point is located in the meridional plane passing through the center of the opening, which corresponds to the major diameter of the elliptical opening, on the basis of the reflection symmetry of the COAM. Thus, let us consider the condition $\mathrm{x}=0$; Eq. (14) is now expressed as follows:

$$
\begin{aligned}
(\sin \theta & \left.\theta\left(u+u_{0}\right)-\cos \theta v+y_{C}\right)^{2} \\
= & 2 R\left(\cos \theta\left(u+u_{0}\right)+\sin \theta v\right) \\
\quad & -(K+1)\left(\cos \theta\left(u+u_{0}\right)+\sin \theta v\right)^{2} .
\end{aligned}
$$

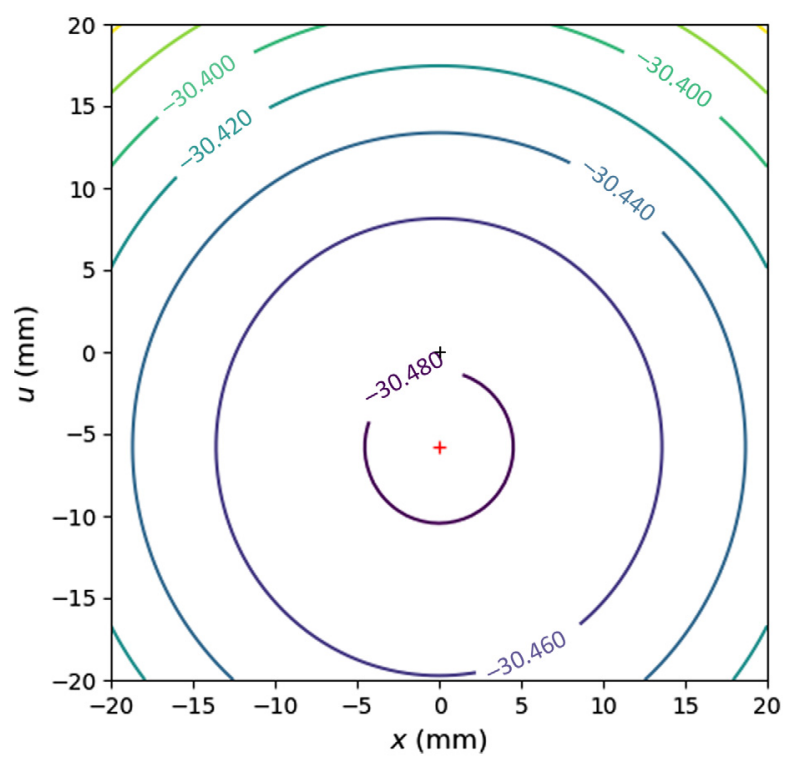

Fig. 4 Contour plot of the depth profile near the opening center for COAM of $R=4000 \mathrm{~mm}, K=-0.7, y_{\mathrm{C}}=439.4 \mathrm{~mm}$, and $\theta=74.45$ deg.
To find the exact location of the deepest point, it is useful to differentiate Eq. (16) to obtain as follows:

$$
\begin{aligned}
&(\sin \theta\left.\left(u+u_{0}\right)-\cos \theta v+y_{C}\right)(\sin \theta(d u)-\cos \theta d v) \\
&= R(\cos \theta(d u)+\sin \theta d v)-(K+1)\left(\cos \theta\left(u+u_{0}\right)\right. \\
&\quad+\sin \theta v)(\cos \theta(d u)+\sin \theta d v)
\end{aligned}
$$

where $d u$ and $d v$ are the differentials along the $u$ - and $v$-axis, and we obtain as follows:

$$
\begin{aligned}
& \frac{d v}{d u} \\
& =\frac{\left(K \cos ^{2} \theta+1\right)\left(u+u_{0}\right)+y_{C} \sin \theta-R \cos \theta+K \sin \theta \cos \theta v}{+R \sin \theta-K\left(\cos \theta\left(u+u_{0}\right)+\sin \theta v\right) \sin \theta-v+y_{C} \cos \theta} .
\end{aligned}
$$

The condition for the existence of the deepest depth corresponds to the numerator of Eq. (18) being equal to zero; that is,

$\left(K \cos ^{2} \theta+1\right)\left(u+u_{0}\right)+y_{C} \sin \theta-R \cos \theta+K \cos \theta \sin \theta v$ $=0$.

Thus, solving the coupled equations of Eqs. (17) and (19) simultaneously results in a quadratic equation for $\left(u+u_{0}\right)$ as follows:

$a\left(u+u_{0}\right)^{2}-2 b\left(u+u_{0}\right)+c=0$,

where

$\left\{\begin{array}{l}a=\frac{(K+1)\left(K \cos ^{2} \theta+1\right)}{(K \sin \theta \cos \theta)^{2}} \\ b=\left((K+1) y_{C} \sin \theta-R \cos \theta\right) \frac{\left(K \cos ^{2} \theta+1\right)}{(K \sin \theta \cos \theta)^{2}} \\ c=\frac{y_{C}^{2}\left(K \cos ^{2} \theta+1\right)(K+1) \sin ^{2} \theta-2 R\left(K \cos ^{2} \theta+1\right) \sin \theta \cos \theta y_{C}+R^{2} \cos ^{2} \theta\left(1-K \sin ^{2} \theta\right)}{(K \sin \theta \cos \theta)^{2}}\end{array}\right.$.

Among the two solutions of Eq. (20), the correct solution is as follows:

$u+u_{0}=\frac{b-\sqrt{b^{2}-a c}}{a}$.

Because $\sqrt{b^{2}-a c}=-\frac{R \sqrt{K \cos ^{2} \theta+1}}{K \sin \theta \cos \theta}$ (negated because of $-K$ ), Eq. (22) can be simplified as follows:

$u+u_{0}=R \frac{\cos \theta}{K+1}\left(1+\frac{K \sin \theta}{\sqrt{K \cos ^{2} \theta+1}}\right)-y_{C} \sin \theta$.

If the first equation of Eq. (13) is used, Eq. (23), the exact location for the deepest point with respect to the center of the elliptical opening can be expressed as follows:

$$
u^{*}=\frac{K \cos \theta \sin \theta}{K \cos ^{2} \theta+1}\left(R \frac{\sqrt{K \cos ^{2} \theta+1}-\sin \theta}{K+1}-y_{C} \cos \theta\right)
$$


and the corresponding depth as follows:

$v^{*}=R \frac{\sin \theta-\sqrt{K \cos ^{2} \theta+1}}{K+1}+y_{C} \cos \theta$.

The numerical depth profile for the COAM shown in Fig. 2 has its deepest point $5.8 \mathrm{~mm}$ from the center of the opening, and the maximum depth is $-30.4825 \mathrm{~mm}$.

In short, the deepest point of a COAM is located at a different point from the center of the elliptical opening when the COAM was obtained by using a plane to cut the aspheric surface, indicating that the reference point for the distance from the center of the opening from the ROA can be either the center of the opening or the deepest point of the mirror. The distance from the ROA to the reference point is called the $Y$-offset or the offaxis distance (OAD). We chose to call the distance the OAD. For both cases, the major diameter of the elliptical opening will be called the clear aperture (CA), which is equal to the size of the COAM. Thus, in the following two sections, we will investigate the details of the COAM for both cases.

\section{First Method of Defining OAD for COAM}

In the first method, the OAD refers to the deepest point of the mirror, as shown in Fig. 5, and the coordinate of the deepest point is $\left(0, \mathrm{OAD}, z_{\mathrm{OAD}}\right)$, where

$z_{\mathrm{OAD}}=\frac{R-\sqrt{R^{2}-(K+1) \mathrm{OAD}^{2}}}{K+1}$

Because the tangent plane at the deepest point is parallel to the $x u$-plane $\left(\left.\because \frac{d v}{d u}\right|_{\left(u^{*}, v^{*}\right)}=0\right)$, the plane cutting the aspheric surface must be parallel to the tangential plane, indicating that the angle for the plane can be obtained by the angle of the tangent plane at the OAD. To obtain the angle, Eq. (2) can be differentiated as follows:

$\frac{d y}{d z}=\frac{R-(K+1) z}{y}$,

and by using $y=\mathrm{OAD}$ and Eq. (26), the angle of the plane can be obtained as follows:

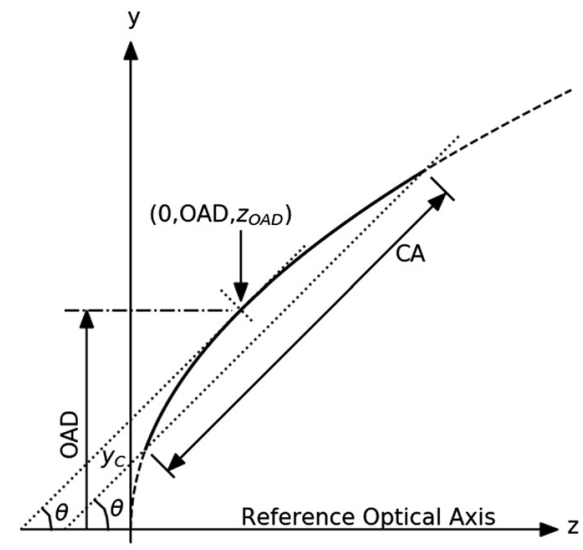

Fig. 5 First method of defining OAD. $\tan \theta=\sqrt{\left(\frac{R}{\mathrm{OAD}}\right)^{2}-(K+1)}$.

Because the desired size of the COAM is equal to the major diameter of the ellipse, denoted by $\mathrm{CA}$, the right-hand side of the third equation of Eq. (6) can be expressed as follows:

$\mathrm{CA}=2 \frac{\cos \theta}{\left(K \cos ^{2} \theta+1\right)} \sqrt{R^{2}-2 R \tan \theta y_{C}-y_{C}^{2}(K+1)}$.

By combining Eqs. (28) and (29), the $y$-intercept, $y_{C}$, for the plane can be obtained, after some algebraic work of rearranging and regrouping the terms, as follows:

$y_{C}$

$$
=\frac{-R \sqrt{\left(\frac{R}{\mathrm{OAD}}\right)^{2}-(K+1)}+\left(\frac{R}{\mathrm{OAD}}\right) \sqrt{R^{2}-\frac{(K+1) C A^{2}}{4\left(\left(\frac{R}{\mathrm{OAD}}\right)^{2}-K\right)}\left(\frac{R}{\mathrm{OAD}}\right)^{2}}}{(K+1)} .
$$

Once the aperture plane is determined, the relative location of the deepest point with respect to the center of the opening, after substituting Eqs. (28) and (30) into Eq. (25), can be obtained as follows:

$$
=\frac{K(\mathrm{OAD}) \sqrt{R^{2}-(K+1)(O A D)^{2}}\left(1-\sqrt{1-\frac{(K+1)(\mathrm{CA})^{2}}{4\left(R^{2}-K(O A D)^{2}\right)}}\right)}{(K+1) \sqrt{R^{2}-K(O A D)^{2}}} .
$$

For the segment $\mathrm{OAD}=1100 \mathrm{~mm}$ and $\mathrm{CA}=1026 \mathrm{~mm}$ of the aspheric surface with $R=4000 \mathrm{~mm}$ and $K=-0.7$ as an example, the numerical values of the angle and the $y$-intercept of the aperture plane are $\theta=74.4548 \mathrm{deg}$ and $y_{\mathrm{C}}=$ $439.41 \mathrm{~mm}$. Figure 6 shows the depth profiles numerically

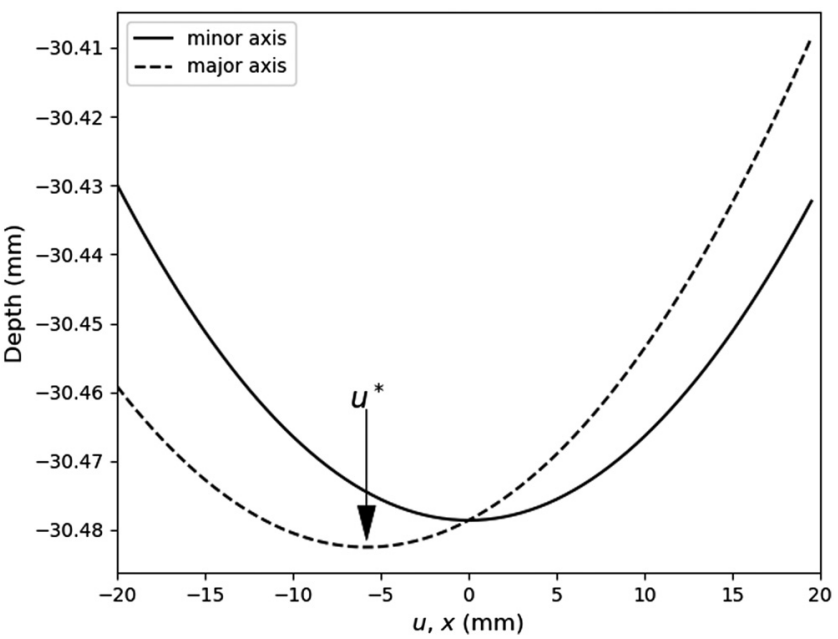

Fig. 6 Depth profile in two orthogonal directions for the segment defined by the first method with the set of parameters $R=$ $4000 \mathrm{~mm}, K=-0.7, \mathrm{OAD}=1000 \mathrm{~mm}$, and $\mathrm{CA}=1026 \mathrm{~mm}$. 
computed for both orthogonal directions for the segment mentioned above. The blue line corresponds to the depth profile along the major axis, and the red line corresponds to the depth profile along the minor axis near the center of the aperture. Along the major axis, the depth profile clearly shows a minimum at $u^{*}=-5.80094 \mathrm{~mm}$ from the center of the aperture as computed by Eq. (31), and the depth of $-30.4825 \mathrm{~mm}$ is $0.0039 \mathrm{~mm}$ deeper than the depth at the center of the aperture.

\section{Second Method of Defining OAD and CA for an Off-Axis Aspheric Segment}

The second method of specifying an off-axis aspheric segment is to define the OAD as the distance from the ROA to the center of the aperture, as shown in Fig. 7. The CA in this case is similarly defined as the major diameter of the elliptical aperture. Unlike in the first method, the angle of the aperture plane is not known a priori. Thus, both the angle and the $y$-intercept of the aperture plane must be solved by applying the two conditions of the OAD and CA to Eq. (7) as follows:

$$
\left\{\begin{array}{l}
\mathrm{OAD}-y_{C}=\sin \theta u_{0} \\
\mathrm{CA}=2 R_{u}
\end{array}\right.
$$

or

$$
\left\{\begin{array}{l}
\mathrm{OAD}=\frac{R \cos \theta \sin \theta+(K+1) \cos ^{2} \theta y_{C}}{K \cos ^{2} \theta+1} \\
\mathrm{CA}=\frac{2 \cos \theta}{K \cos ^{2} \theta+1} \sqrt{R^{2}-2 R \tan \theta y_{C}-(K+1) y_{C}^{2}}
\end{array}\right.
$$

Equation (33) is a coupled equation of the two parameters of $\mathrm{CA}$ and OAD in terms of $\theta$ and $y_{\mathrm{C}}$ and can be reversed to solve for the parameters of $\theta$ and $y_{C}$ in terms of CA and OAD. The first equation of Eq. (33) was rewritten to make the variable $y_{\mathrm{C}}$ a function of OAD:

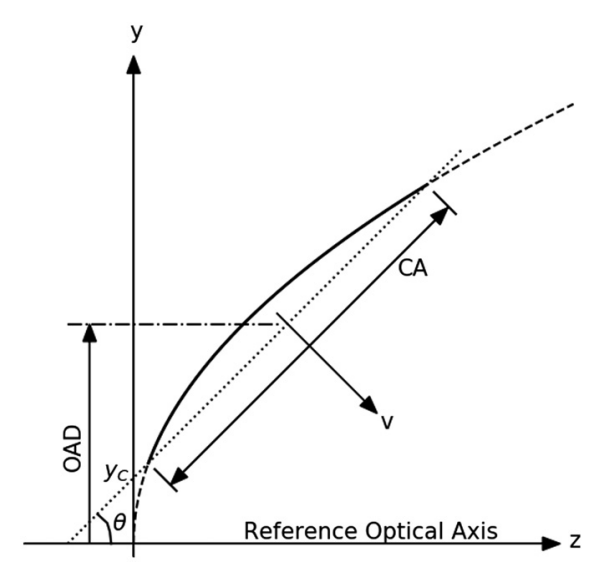

Fig. 7 Second method of defining OAD.
$y_{C}=\frac{(\mathrm{OAD})\left(K \cos ^{2} \theta+1\right)-R \cos \theta \sin \theta}{(K+1) \cos ^{2} \theta}$.

Now, Eq. (34) was substituted into the second equation of Eq. (33) to obtain a quadratic equation with respect to the variable $m^{2}$ after some trivial algebra:

$a m^{4}+b m^{2}+c=0$,

where

$m=\tan \theta$,

and

$\left\{\begin{array}{l}a=4(\mathrm{OAD})^{2} \\ b=4(\mathrm{OAD})^{2}(K+2)+(\mathrm{CA})^{2}(K+1)-1 R^{2} \\ c=(\mathrm{CA})^{2}(K+1)^{2}-1 R^{2}+4(\mathrm{OAD})^{2}(K+1)\end{array}\right.$.

Unfortunately, the term $b^{2}-4 a c$ for Eq. (37) cannot be further reduced to the square of a sum, and no further simplification is possible. Among the two possible solutions of the quadratic equation, the positive value is the correct solution because its square root has to be taken to calculate the final solution for $m$. Once the angle and the $y$-intercept are obtained, the location and depth for the deepest point can be calculated by using Eqs. (24) and (25).

\section{Comparison of Two Methods and Discussion}

Table 1 shows a list of characteristics of the COAM defined by the two different methods with the same values of OAD and CA and the radius of curvature, and conic constant. The numeric values indicate only a small difference between the two methods. However, the difference of $\sim 10 \mu \mathrm{m}$ in the maximum depth cannot be ignored when the requirements of tolerance in the values of $\mathrm{CA}$ and $\mathrm{OAD}$ are tight.

The methods will help define a COAM with an aperture of a plane, unlike those defined by the projection. It is easy to distinguish the center of the aperture, the deepest point of the mirror, and the distance between the two points. Furthermore, several equations can directly yield their values. Prior to these methods, one must complete a series of computations from the sag equation with various conditional check points to ensure that the computations are done properly.

Most importantly, it is noteworthy to understand two methods of a very similar COAM. To demonstrate the difference between the two methods, various characteristics are evaluated as a function of a conic constant while other parameters remain constant. The differences between the two methods are shown in Fig. 8. As the conic constant approaches -1, the difference between the two methods disappears. The disappearing

Table 1 Comparison the values calculated by two definitions for the OAD with the set of parameters $R=4000 \mathrm{~mm}, K=-0.7, \mathrm{OAD}=1100 \mathrm{~mm}$, and $\mathrm{CA}=1026 \mathrm{~mm}$.

\begin{tabular}{lllccc} 
Method & $\theta(\mathrm{deg})$ & $y_{\mathrm{C}}(\mathrm{mm})$ & $u^{*}(\mathrm{~mm})$ & Maximum depth $(\mathrm{mm})$ & Depth at $u_{0}(\mathrm{~mm})$ \\
\hline 1 & 74.4548 & 439.414 & -5.80094 & 30.4825 & 30.4786 \\
2 & 74.4193 & 441.023 & -5.81219 & 30.4717 & 30.4678 \\
\hline
\end{tabular}



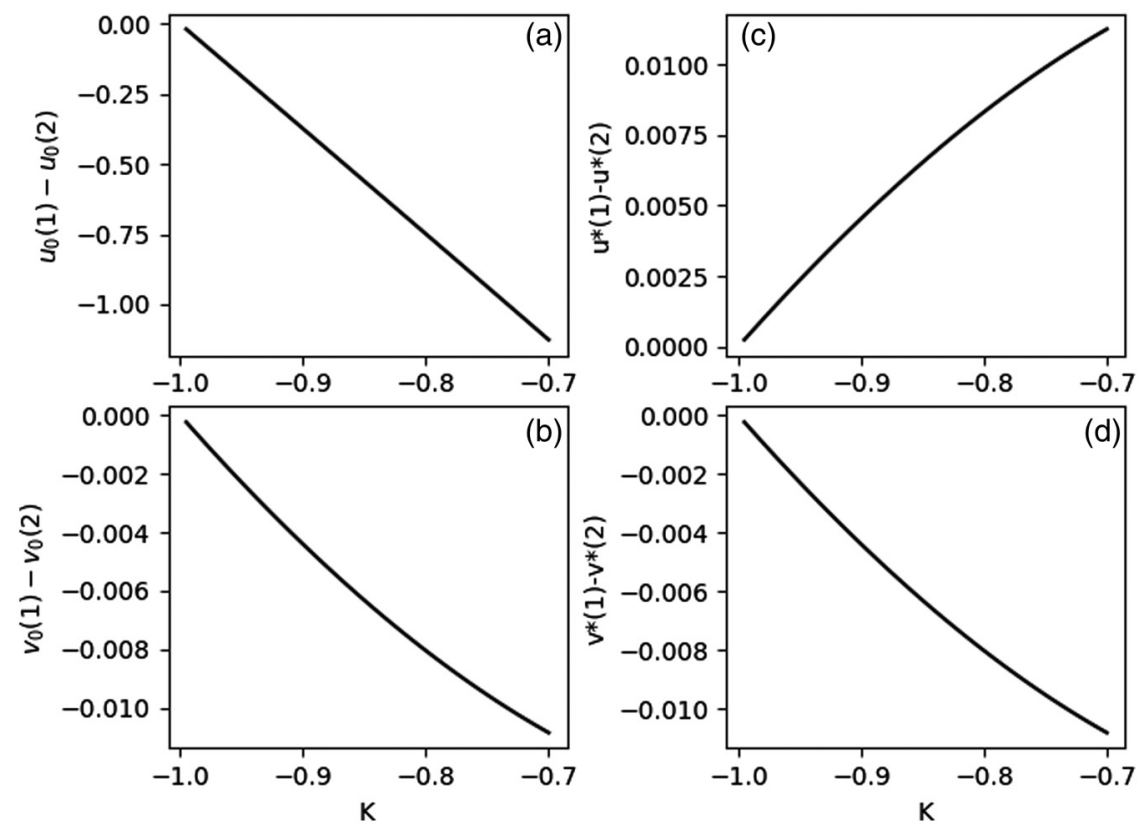

Fig. 8 Differences between two methods for various characteristics of (a) $u_{0}$, (b) $v_{0}$, (c) $u^{*}$, and (d) $v^{*}$ for a set of same parameters of $R=4000 \mathrm{~mm}, \mathrm{OAD}=1100 \mathrm{~mm}$, and CA $=1026 \mathrm{~mm}$.

differences confirm the correct evaluation because the two methods become equivalent for off-axis parabolic mirrors. ${ }^{11}$ The monotonic trend of various characteristics also indicates that small differences are not artifacts or errors caused by the computations. They instead indicate the nature of the COAM defined by the two methods.

The second method seems more intuitive because of the center of the aperture. However, because the angle of the aperture plane can be obtained directly, the first method might be more reasonable. More studies on the difference between the two methods with respect to the fabrication and testing COAMs should be performed.

\section{Summary}

We presented a thorough analysis of an off-axis aspheric segment considered as a part of an aspheric surface cut by a plane. We found that the off-axis segment is an elongated ellipse along the direction of the meridional plane and that the deepest point is different from the center of the aperture of the segment. This indicates that we should trace the location of contact point during all grinding processes because the location of the point is not fixed. This is not trivial for both fabrication and testing points of view. The difference also led us to consider two different methods of defining the so-called OAD. The two methods are slightly different from each other in every aspect of the segment.

Every telescope shares a series of common procedures for designing, optimizing, manufacturing, and testing the segment over a long period of time, ${ }^{12}$ and the development of an unambiguous definition of a segment cannot be overemphasized. When a telescope consists of multiple segments, each of which needs to be manufactured at different sites, such a definition is essential. It is highly desirable that the standard include correspondingly clear definitions. The definitions presented here, we believe, can minimize guesswork and uncertainty and improve the repeatability of multiple segment production.

\section{Acknowledgments}

This research was supported by the Korea Astronomy and Space Science Institute under the R\&D program supervised by the Ministry of Science, ICT, and Future Planning, Daejeon, Republic of Korea and in part by the Inha University, Incheon, Republic of Korea, and in part by the Korea Basic Science Institute, Daejeon, Republic of Korea. The authors thank anonymous reviewers for their valuable comments.

\section{References}

1. R. A. Bernstein et al., "Overview and status of the Giant Magellan Telescope project," Proc. SPIE 9145, 91451C (2014).

2. J. H. Burge et al., "Design and analysis for interferometric measurements of the GMT primary mirror segments," Proc. SPIE 6273, 62730M (2006).

3. TMT Observatory Corporation, "Draft statement of work for production of the TMT primary mirror optics," http://citeseerx.ist.psu.edu/viewdoc/ download;jsessionid=665E0636E52C383B56934F30C1B66786?doi= 10.1.1.434.5303\&rep=rep1\&type=pdf (2009).

4. M. Cayrel, "E-ELT Optomechanics: Overview," Proc. SPIE 8444, 84441X (2012).

5. H. P. Stahl, "Testing the James Webb Space telescope primary mirror," https://ntrs.nasa.gov/archive/nasa/casi.ntrs.nasa.gov/20140008581.pdf.

6. C. J. Oh et al., "Fabrication and testing of $4.2 \mathrm{~m}$ off-axis aspheric primary mirror of Daniel K. Inouye solar telescope," Proc. SPIE 9912, 991200 (2016).

7. J.-Y. Han and S. Lee, "Methods to define circular off-axis aspheric segment mirrors," Proc. SPIE 10742, 107421I (2018).

8. Y. S. Kim et al., "Prototype development for the GMT FSM secondaryoff-axis aspheric mirror fabrication," J. Astron. Space Sci. 31(4), 341-346 (2014)

9. J. N. Choi et al., "Integrated ray tracing simulation of the SCOTS surface measurement test for the GMT fast steering mirror prototype," Adv. Space Res. 56(11), 2483-2494 (2015). 
10. G. Cavli, "Shortest distance between two skew lines," http://www .physicsandmathstutor.com/a-level-maths/shortest-distance-two-skewlines/ (2014).

11. J.-Y. Han and S. Lee, "Geometry for off-axis parabolic mirrors," Proc. SPIE 10706, 107066M (2018).

12. S. E. Kendrick and H. P. Stahl, "Large aperture space telescope mirror fabrication trades," Proc. SPIE 7010, 70102G (2008).
Jeong-Yeol Han is a principle researcher at Korea Astronomy and Space Science Institute. He received his $\mathrm{PhD}$ degree in astronomy and space science from the University of Science and Technology in 2008. His current research interests include optical systems development, polishing process control, and optical testing.

Biographies for the other authors are not available. 